\title{
BMJ Open Dog ownership and cardiovascular risk factors: a nationwide prospective register-based cohort study
}

\author{
Mwenya Mubanga, ${ }^{1}$ Liisa Byberg, ${ }^{2}$ Agneta Egenvall, ${ }^{3}$ Johan Sundström, ${ }^{4}$ \\ Patrik Karl Erik Magnusson, ${ }^{5}$ Erik Ingelsson, ${ }^{1,6}$ Tove Fall ${ }^{\oplus}$
}

To cite: Mubanga M, Byberg L, Egenvall $A$, et al. Dog ownership and cardiovascular risk factors: a nationwide prospective register-based cohort study. BMJ Open 2019;9:e023447. doi:10.1136/ bmjopen-2018-023447

- Prepublication history and additional material for this paper are available online. To view these files, please visit the journal online (http://dx.doi. org/10.1136/bmjopen-2018023447).

The results of this study were previously presented as a poster at the World Congress or Cardiology and Cardiovascular Health 2018 and an abstract was published in Global Heart Volume 13, Issue 4, December 2018, Page 483.

Received 9 April 2018

Revised 1 November 2018

Accepted 17 January 2019

Check for updates

(C) Author(s) (or their employer(s)) 2019. Re-use permitted under CC BY-NC. No commercial re-use. See rights and permissions. Published by BMJ.

For numbered affiliations see end of article.

Correspondence to

Dr Tove Fall;

tove.fall@medsci.uu.se

\section{ABSTRACT}

Objective To study the association between dog ownership and cardiovascular risk factors.

Design A nationwide register-based cohort study and a cross-sectional study in a subset.

Setting A cohort of 2026865 participants was identified from the Register of the Total Population and linked to national registers for information on dog ownership, prescribed medication, hospital admissions, education level, income and country of birth.

Participants were followed from 1 0ctober, 2006, to the end of the study on 31 December, 2012, assessing medication for a cardiovascular risk factor, emigration and death. Cross-sectional associations were further assessed in 10110 individuals from the TwinGene study with additional adjustment for professional level, employment status, Charlson comorbidity index, disability and tobacco use.

Participants All Swedish residents aged 45-80 years on 1 October, 2006.

Main outcome measures Initiation of medication for hypertension, dyslipidaemia and diabetes mellitus.

Results After adjustment for confounders, the results indicated slightly higher likelihood of initiating antihypertensive (HR, 1.02; 95\% Cl, 1.01 to 1.03$)$ and lipid-lowering treatment ( $\mathrm{HR}, 1.02 ; 95 \% \mathrm{Cl}, 1.01$ to 1.04$)$ in dog owners than in non-owners, particularly among those aged 45-60 years and in those owning mixed breed or companion/toy breed dogs. No association of dog ownership with initiation of treatment for diabetes was found in the overall analysis ( $\mathrm{HR}, 0.98 ; 95 \% \mathrm{Cl}, 0.95$ to 1.01). Sensitivity analyses in the TwinGene cohort indicated confounding of the association between dog ownership and prevalent treatment for hypertension, dyslipidaemia and diabetes mellitus, respectively, from factors not available in the national cohort, such as employment status and non cardiovascularchronic disease status.

Conclusions In this large cohort study, dog ownership was associated with a minimally higher risk of initiation of treatment for hypertension and dyslipidaemia implying that the previously reported lower risk of cardiovascular mortality among dog owners in this cohort is not explained by reduced hypertension and dyslipidaemia. These observations may suffer from residual confounding despite access to multiple important covariates, and future studies may add valuable information.

\section{Strengths and limitations of this study}

This is the largest study to date to examine the impact of dog ownership on cardiovascular risk factors.

- The nationwide register-based cohort study with a cross-sectional investigation in a twin registry with a vast array of lifestyle and clinical variables strengthens the results.

- The main outcome measures were extracted from nationwide registers thus decreasing the risk of recall and selection bias.

- Misclassification of dog ownership, particularly in the twin register, may have led to some loss of power.

- Some important confounding factors were not available in the national data.

\section{INTRODUCTION}

There is a growing interest in pet ownership as a possible intervention to enhance cardiovascular health and well-being. ${ }^{12}$ We recently observed that being registered as a dog owner was associated with a lower risk of cardiovascular and all-cause mortality in the general Swedish population $(n=3432153){ }^{3}$ Any causal association of dog ownership with lower cardiovascular mortality could potentially be mediated through increased physical activity ${ }^{45}$ or through the psychological benefits of companionship, ${ }^{6}$ which could in turn reduce other important cardiovascular risk factors such as blood pressure, adiposity, dyslipidaemia and insulin resistance. ${ }^{78}$ An alternative explanation could be confounding by socioeconomic, ${ }^{9}$ cultural,${ }^{10}$ demographic ${ }^{9}$ or psychosocial factors. ${ }^{11}{ }^{12}$ A large number of cross-sectional and longitudinal studies across different countries support the association of dog ownership with physical activity, ${ }^{1}$ however, reports regarding the association of dog ownership with other cardiovascular risk factors are less consistent. ${ }^{13-20}$ These inconsistencies may be due to low statistical power in small studies, use of restricted or 
homogenous populations, inability to control for differences across breeds of dogs, or simply an absence of effect. As dogs are reported to be more common in rural areas compared with urban areas, ${ }^{21-23}$ as well as in households with children, ${ }^{24}{ }^{25}$ it is also important to account for these differences. The aim of this study was to assess the association of dog ownership with three major clinical risk factors for cardiovascular disease (CVD),${ }^{26}$ specifically initiation of treatment of hypertension, dyslipidaemia and diabetes mellitus. We hypothesised that the cardiovascular risk profile of dog owners is better than that of non-dog owners. To overcome limitations of previous studies concerning study size, generalisability and differences between dog breeds, we investigated this hypothesis using data on all Swedish residents aged 45-80 years of age in 2006 from national registers on dog ownership and drug prescriptions. We further sought to explore the association with other cardiovascular risk factors using cross-sectional data from a subcohort extracted from the Swedish Twin Registry containing detailed information from questionnaire data, physical examinations and laboratory measurements.

\section{METHODS}

\section{Design}

The main analysis was based on a nationwide cohort study of Swedish residents aged 45-80 followed from 1 October 2006 to 31 December, 2012. We additionally used cross-sectional data of participants (aged 47-80 years) in the TwinGene study, which is a substudy of the Swedish Twin Registry (see online supplementary figure 1).

\section{Study population-national cohort}

All Swedish residents ( $n=3$ 412946) aged $45-80$ years on 1 October, 2006, were identified through the Register of the Total Population. To ensure complete linkage to medical information and sufficient information regarding dog ownership in Sweden, we excluded 11298 individuals with unverified, reused identification numbers or missing education information, and 137306 additional individuals that had resided in Sweden for $<15$ years. We also excluded 531658 individuals with a history of any CVD (International Classification of Disease [ICD]-9 codes 390-459 and ICD-10 I00-I99) before 1 October, 2006 or with a history of coronary artery bypass grafts or percutaneous coronary artery intervention medical procedure (Nordic surgical procedure codes FNA (anastomosis of internal thoracic artery with coronary arteries), FNC (aorto-coronary bypass using vein graft) and FNG (dilatation and recanalization of coronary arteries) from inpatient and outpatient data. Inpatient data were available from 1987 and outpatient data from 2001. Further, using data from the Swedish Prescribed Drug Register, which covers all Swedish dispensed pharmacy prescriptions since it was established on 1 July, 2005, individuals $(\mathrm{n}=705819)$ were excluded if they had any recorded dispensed prescription of antihypertensive drugs, lipid-lowering drugs or glucose-lowering drugs from 15 months prior to baseline (which was when this register was initiated). Antihypertensive drugs were defined based on the Anatomical Therapeutic Chemical Classification System (ATC) as codes: C02 (antihypertensive drugs), C03A, C03EA01 (thiazide diuretics), C07 (beta-receptor blockers, excluding sotalol [C07AA07]), C08C (selective calcium antagonists with mainly vascular effects) and C09 (agents acting on the renin-angiotensin system). Lipid-lowering drugs were defined as C10AA (statins), C10AB (fibrates), C10AC (bile acid sequestrants), C10AX (other lipid-modifying agents) and C10B (lipid-lowering drug combinations). Glucose-lowering drugs were defined as ATC-code A10A (insulin and analogues) and A10B (glucose-lowering drugs excluding insulin).

\section{Study population-TwinGene}

The TwinGene study originally included 12614 (of 22391 invited) twins from the 'Screening Across the Lifespan Twin study' (SALT). It was conducted between April 2004 and December 2008 and included a visit to the participants' local health centre and blood sampling (see online supplementary figure 2). ${ }^{27}$ The study-base 'SALT' was a substudy of the Swedish Twin Register in twins born before 1959 and who participated in a telephone-based questionnaire substudy from March 1998 to March $2002^{27}$ (see online supplementary table 1).

We performed a cross-sectional analysis of the association of dog ownership with cardiovascular risk factors in the TwinGene cohort $(\mathrm{n}=12105)$. We excluded 1373 individuals for having a previous history of CVD recorded in the National Patient Register. ${ }^{28}$ We also excluded 622 individuals for having missing or incomplete data (see online supplementary figure 1 ).

\section{Exposure}

Dogs in Sweden are required to have a unique identifier (ear tattoo or implanted identity chip) and this is registered alongside their owner's unique personal identity number at the Swedish Board of Agriculture. All dogs sold as purebred are registered by the Swedish Kennel Club. In Sweden, there are virtually no stray $\operatorname{dogs},{ }^{29}$ and compliance to regulations is thought to be high due to a general high level of social and institutional trust. ${ }^{30}$

We defined the variable 'dog ownership' in the national cohort as registered dog ownership or having a partner registered as a dog owner in either the Swedish Board of Agriculture and/or the Swedish Kennel Club registers. Exposure to dog ownership was time updated to include only those periods where each dog was alive and registered to the study participant or their registered partner. The identification of partners was possible through annual extracts from the Register of the Total Population that keeps track of couples that are married, registered in same-sex partnership or are cohabiting with common children. It is presently not possible to identify cohabiting non-married partners who have no children in common in the population registers. 


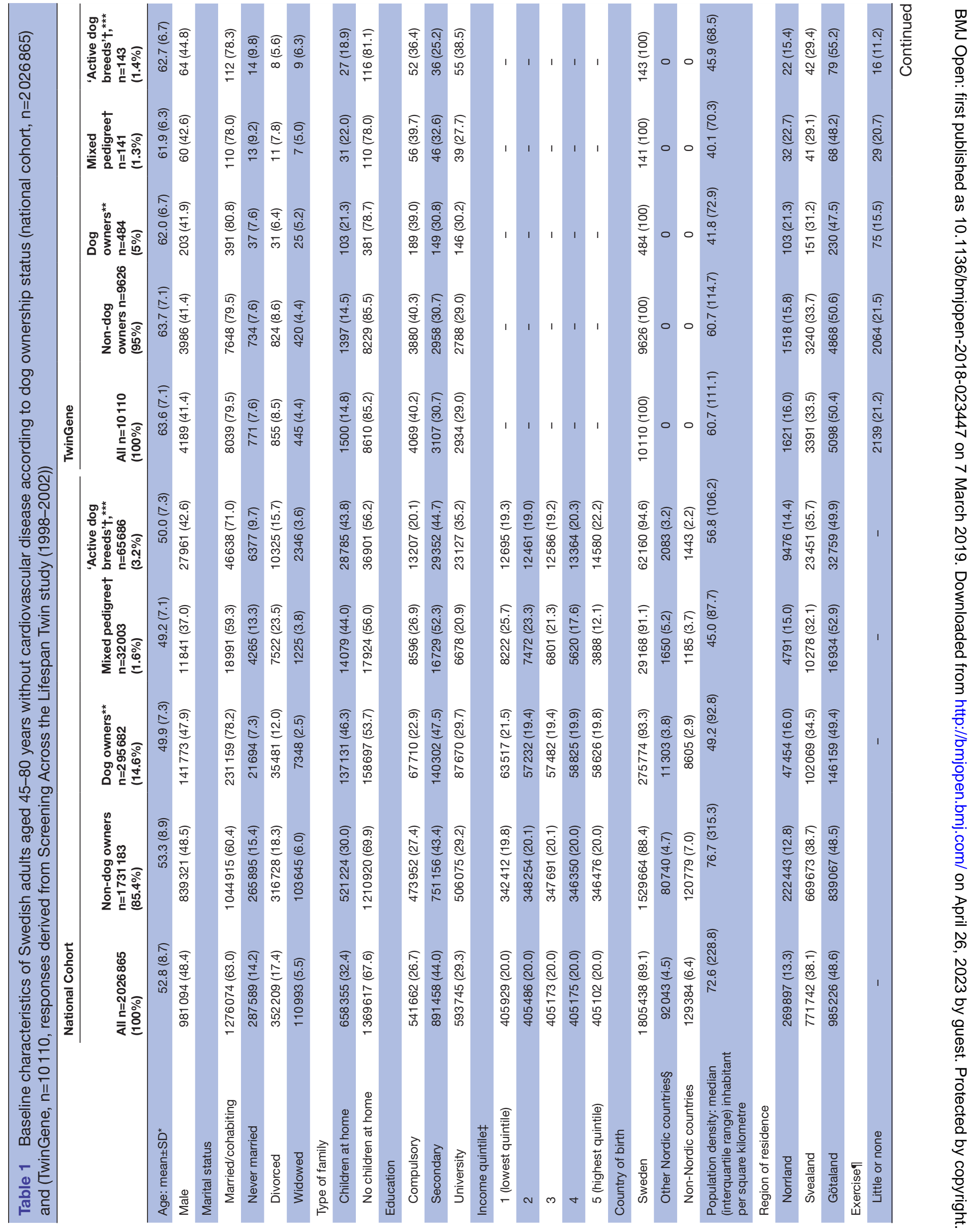


In the TwinGene data, we did not have access to information on partners' dog ownership and only each person's own dog registrations were used. Dog ownership was defined at the date of inclusion in TwinGene.

If information on a dog's death was missing, we assumed a maximum lifespan of 10 years. ${ }^{31}$ We conducted sensitivity analyses examining associations with dog death at a maximum lifespan of 8 years and 12 years. Where birth or registration dates were discrepant between the two registers, we randomly selected one of the two.

To define breed groups, we used the Federation Cynologique International standard with some local adaption from Swedish Kennel Club's definition to categorise the 331 breeds into 10 breed groups based on character and behaviour attributes (see online supplementary table 2). All non-purebred dogs and those of unknown breed were included in an additional mixed-breed group. Where owners had dogs of different breeds, we defined the breed based on the dog registered first and where owners had several dogs, we restricted ownership to three dogs.

Based on previous findings ${ }^{3}$ that ownership to four different breed groups was associated with a lower risk of cardiovascular events, we defined a group of these dog breeds (terriers, pointing, scent hounds and retrievers) for additional exploratory analysis. This group is hereafter referred to as 'active dog breeds' as these breeds also generally demand high levels of physical activity.

\section{Outcome}

In the national cohort, time to first dispensed prescription of antihypertensive drugs, lipid-lowering drugs or glucose-lowering drugs after baseline was defined from data extracted from the drug register. Each outcome was considered separately as we chose to estimate the total effect of dog ownership and not only the direct effects. Participants were censored at emigration, death or at the end of the study on 31 December, 2012. In the analysis of time to antihypertensive medication, individuals were additionally censored at a diagnosis of heart failure, unstable angina or acute myocardial infarction in the National Patient Register as the same drugs could be administered for their treatment.

Prevalent use of antihypertensive, lipid-lowering or glucose-lowering drugs was defined from the clinical questionnaire data collected during the TwinGene study. Cardiovascular risk factors measured and also used as outcomes in TwinGene included blood glucose, glycosylated haemoglobin Alc, high sensitive C reactive protein (hsCRP), triglycerides, high-density lipoprotein-cholesterol, low-density lipoprotein-cholesterol, waist-hip ratio, body mass index (BMI), systolic and diastolic blood pressure and mean arterial pressure (see online supplementary methods). Only fasting measurements of glucose and triglycerides were used (9873 [97\%] of all participants were fasting). 
Table 2 Association of dog ownership with initiation of medication for hypertension, dyslipidaemia and diabetes

\begin{tabular}{|c|c|c|c|c|c|c|}
\hline Cohort & Medication & $\mathrm{N}$ treated & Time at risk & Model 1* & Model 2† & Model 3‡ \\
\hline \multirow[t]{2}{*}{ National } & Hypertension & 503305 & 10659258 & $1.02(1.01$ to 1.03$)$ & 1.02 (1.01 to 1.03$)$ & NA \\
\hline & Dyslipidaemia & 276691 & 11508349 & $1.03(1.02$ to 1.04$)$ & $1.02(1.01$ to 1.04$)$ & NA \\
\hline \multirow[t]{2}{*}{ TwinGene } & Hypertension & 2223 & NA & $0.96(0.75$ to 1.21$)$ & $0.94(0.74$ to 1.20$)$ & $0.90(0.70$ to 1.15$)$ \\
\hline & Dyslipidaemia & 963 & NA & 0.92 (0.65 to 1.29$)$ & 0.92 (0.65 to 1.29$)$ & 0.87 (0.62 to 1.22$)$ \\
\hline
\end{tabular}

For national cohort ( $\mathrm{n}=202$ 6865), Cox regression models with HRs and 95\% Cl for incident medication are applied, while logistic models for prevalent use is used in TwinGene ( $n=10110)$ and ORs presented.

Estimates in bold represents associations with $p<0.05$.

*Model 1. Age and sex adjusted.

†Model 2. National cohort: Adjusted for sex, age, type of family, area of residence, population density, marital status, region of birth (Sweden, Nordic, non-Nordic), income, education level, latitude of residence. TwinGene: adjusted for sex, age, type of family, area of residence, population density, marital status, education level and latitude of residence.

¥Model 3. Adjusted for sex, age, number of children in the home, area of residence, population density, marital status, tobacco use, occupational level, employment status, disability and Charlson comorbidity index.

NA, not applicable.

\section{Statistical analyses}

All statistical analyses were conducted using Stata V. MP14.1 (StataCorp).

Using age as a timescale, separate multivariable Cox proportional hazards models were applied to assess the associations between dog ownership and time to initiation of antihypertensive, lipid-lowering and glucose-lowering drugs, respectively. Directed acyclic graphs were used to guide the choice of covariates (see online supplementary figure 3). A first crude model included age and sex, and a second model additionally included the region of birth, area of residence, latitude of residence, population density, level of education, marital status, presence of children in the home and income. A description of the covariates is provided in the online supplementary methods. The proportional hazards assumption was verified by plotting Schoenfield residuals and log-log graphs. Results were reported as HRs and 95\% CIs. We repeated the calculations using the breed group as exposure to examine possible breed group effects and we applied Bonferroni correction (for 11 breed groups) to control for multiple testing. Further analyses were stratified by age group, sex, and whether participants lived alone or not. Individuals considered as 'living alone' did not have any spouse, partner with common children, or children living in the same household.

We conducted a sensitivity analysis where we excluded $\beta$-blockers as first line antihypertensive treatment to estimate the effect of changing treatment guidelines over the study period. In additional sensitivity analysis, in the lipid-lowering medication analysis, we assessed the effect of censoring participants at a diagnosis of heart failure, unstable angina or acute myocardial infarction in the National Patient Register.

Logistic regression was applied in TwinGene for the association of $\operatorname{dog}$ ownership with prevalent antihypertensive, lipid-lowering and blood-glucose-lowering medication and linear regression for the association of dog ownership with continuous variables. hsCRP and triglycerides were transformed to the natural log scale before analysis to approach normality.

In addition to adjusting for age, sex, presence of children in the household, area of residence, population density, marital status, latitude of residence and level of education, we added further covariates, one at a time to investigate their individual importance: tobacco use, occupational level, employment status, Charlson comorbidity index and disability. In all twin analyses, SEs were adjusted with the robust sandwich estimator for dependent observations. For blood pressure and lipid levels, associations were further stratified by current medication.

\section{Patient involvement}

No patients were involved in the development, design or analysis of this study.

\section{RESULTS \\ National cohort}

The baseline characteristics of 2026865 Swedish residents are shown in table 1 . Dog ownership was directly registered in $189355(9.3 \%)$ at any time during the follow-up period, and this increased to $295682(14.6 \%)$ individuals when partners' registration were included. At baseline, the average age of dog owners was 50 years versus 53 years in non-owners. Dog owners were more likely to be married than non-owners ( $78 \%$ vs $60 \%)$ and more likely to live in low-density areas than non-owners (median: 49 vs 77 inhabitants per square kilometre). Compared to non-owners, mixed pedigree dog owners $(n=32,003)$ were less likely to be married (59\%), were less likely to have a tertiary education $(21 \%)$ and had fewer people in the top quintile for income (12.2\%). Owners of 'active dog 
breeds' showed similar characteristics to the overall dog owners.

\section{Medication for cardiovascular risk factors}

During 10692258 person-years of follow-up, dog ownership was associated with a $2 \%$ higher risk of initiation of antihypertensive drug medication in both crude and multivariable-adjusted analyses (HR, 1.02; 95\% CI, 1.01 to 1.03). During 11508349 person-years of follow-up, there was a $2 \%$ higher risk of initiating lipid-lowering medication in the multivariable-adjusted models (HR, 1.02; 95\% CI, 1.01 to 1.04). During 12207964 person-years of follow-up, there was a lower risk of initiating glucose-lowering drugs in dog owners in minimally adjusted models (HR, 0.91; 95\% CI, 0.89 to 0.94 ), but on multivariable adjustment, the association was attenuated and non-significant (HR, 0.98; 95\% CI, 0.95 to 1.01) (table 2).

Owners of 'companion/toy' breeds and of dogs of mixed pedigree were at higher risk of antihypertensive and lipid-lowering drug initiation compared with non-dog owners (table 3). Owners of the Spitz/primitive breed types and the combined group of 'active dog breeds' breed types had lower risks of initiating glucose-lowering medication ( $\mathrm{HR}, 0.83 ; 95 \% \mathrm{CI}, 0.74$ to 0.93 and HR, $0.92 ; 95 \%$ CI, 0.86 to 0.97 , respectively), while owners of mixed pedigree dogs had higher risk of getting glucose-lowering medication (HR, 1.17; 95\% CI, 1.09 to 1.26 ) (see online supplementary figure 4 ).

There was no difference in strength of association when we excluded $\beta$-blockers as first-line treatment for antihypertension (see online supplementary table 3 ) or when censoring was done in those being investigated for lipid-lowering treatment initiation was made for angina, myocardial infarction or heart failure was conducted (see online supplementary table 4 ).

In age-stratified analysis, there were some evidence of effect modification by age for both antihypertensive and lipid-lowering drugs where an increased risk was observed in those aged below 50 years (HR, 1.04; $95 \% \mathrm{CI}, 1.01$ to 1.08 and HR, 1.10; 95\% CI, 1.04 to 1.15 , respectively), with estimates gradually approaching one with increasing age (figure 1). Inverse associations of dog ownership with glucose-lowering drugs was observed in the lower age groups, in males and those not living alone (HR, 0.89; $95 \% \mathrm{CI}, 0.79$ to 0.99 , HR, $0.95 ; 95 \% \mathrm{CI}, 0.92$ to 0.99 and HR, $0.91 ; 95 \%$ CI, 0.86 to 0.97 , respectively).

\section{TwinGene}

On cross-sectional analysis of 10110 individuals, 484 $(5 \%)$ were registered as dog owners (partners' dogs not included) and their characteristics are described in table 1 and online supplementary table 5. Using similar covariates as in the national cohort, no association of dog ownership was found with prevalent use of antihypertensive drugs (OR, 0.94; 95\% CI, 0.74 to 1.20 ), lipid-lowering drugs (OR, 0.92; 95\% CI, 0.65 to 1.29) or glucose-lowering drugs (OR, $0.90 ; 95 \% \mathrm{CI}, 0.50$ to 1.63 ) (table 2). Upon inclusion of additional covariates, the
Charlson comorbidity index and the employment status were found to be the most influential confounders and the fully adjusted model yielded lower but still non-significant estimates: OR, 0.90 (95\% CI, 0.70 to 1.15$)$ for use of antihypertensive drugs, OR, 0.87 (95\% CI, 0.62 to 1.22) for lipid-lowering drugs and OR, 0.78 (95\% CI, 0.43 to 1.43) for glucose-lowering drugs (see online supplementary table 6 ). We found no association between dog ownership and the other clinical and biochemical cardiovascular risk factors (figure 2).

Sensitivity analyses on changing the maximum lifespan of dogs in the national cohort that had no dates of death to 8 years or 12 years yielded similar results to the maximum of 10 years used in the main analysis (see online supplementary table 7). To provide additional information, the output from the fully adjusted Cox regression models for the association of dog ownership with the initiation of medication for hypertension, dyslipidaemia and diabetes mellitus in the national cohort are included in the supplementary material as online supplementary tables $8-9$, respectively.

\section{DISCUSSION}

In this nationwide study in a population without previous cardiovascular disease, we observed a minimally higher risk of initiation of treatment for hypertension and dyslipidaemia among persons with a dog in their household compared with those without dogs in the household. Associations were most prominent in younger age groups (45-60 years). Owning a dog of mixed pedigree or a dog belonging to the 'companion/toy' breed group was associated with hypertension and dyslipidaemia, while ownership of a dog from the 'Spitz/primitive' breed and the combined group of' active dog breeds', consisting of breeds identified in our previous study (terriers, pointing, scent hounds and retrievers), ${ }^{3}$ was associated with lower risk of treatment for diabetes mellitus. Cross-sectional analyses in 10110 participants from TwinGene showed no association of dog ownership with BMI, waist-to-hip-ratio, blood pressure or biochemical cardiovascular risk factors, and indicated that the association of dog ownership with medication for hypertension, dyslipidaemia and diabetes was confounded by employment status and non-CVDchronic conditions. This suggests that the slightly higher associations observed in the national cohort would potentially be attenuated in the presence of the additional confounders.

That owners of mixed breed and 'companion/toy' breeds, as well as dog owners in younger age groups, had mildly increased risks for hypertension and dyslipidaemia are in line with our previous study regarding higher risk of myocardial infarction and stroke in this group. ${ }^{3}$ The level of dog walking might be lower in the smaller companion/toy dogs and mixed-breed dogs as compared with the 'active dog breeds' group, which consists of dog breeds originally bred for hunting. ${ }^{32}$ This was also supported by data from TwinGene where $69.9 \%$ of active 


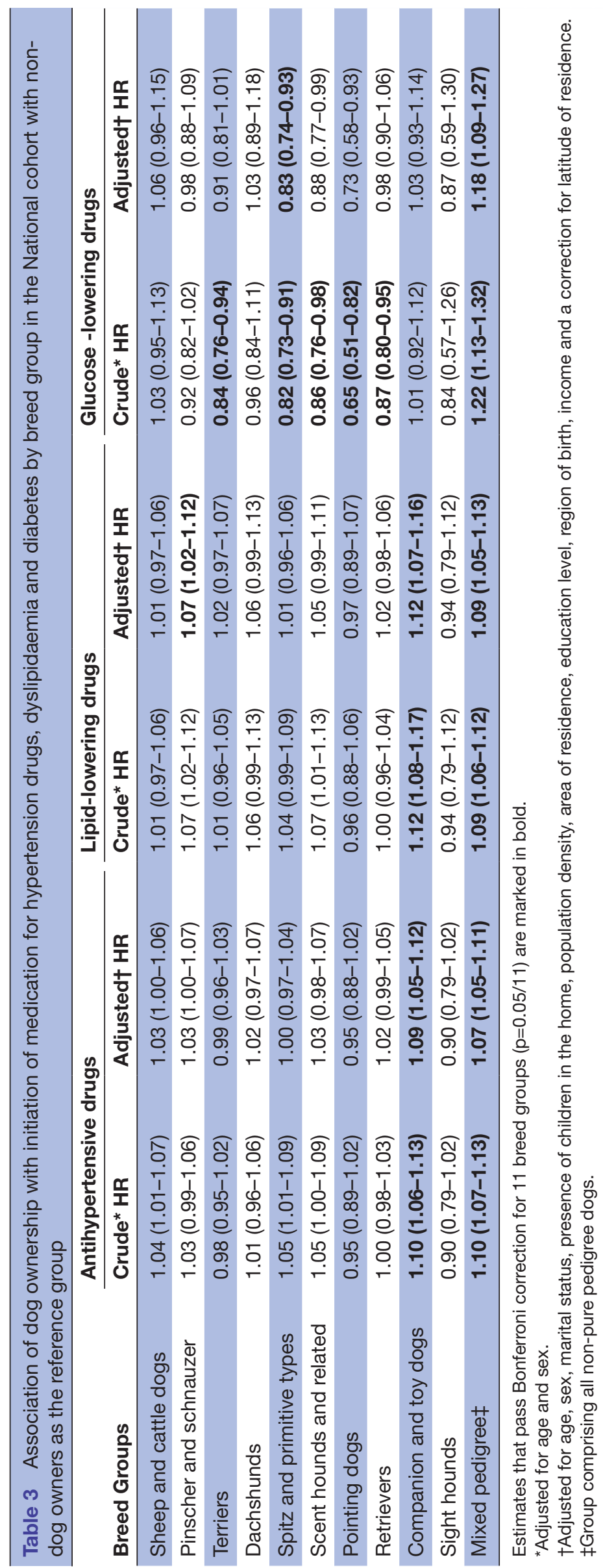




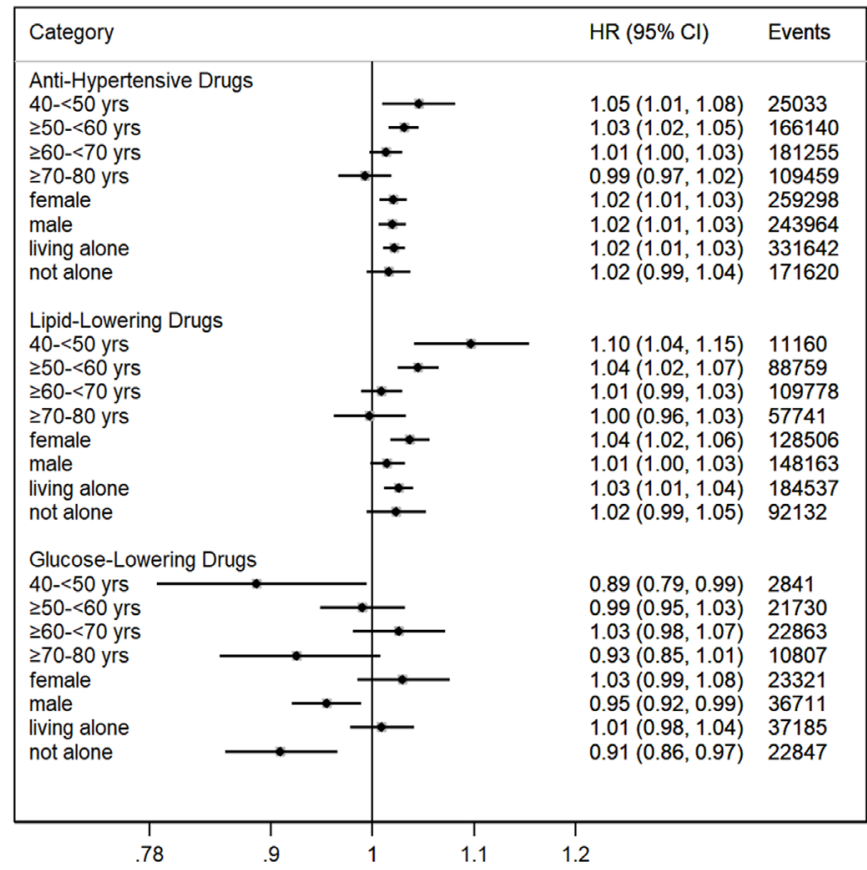

Figure $1 \mathrm{HRs}$ and $95 \% \mathrm{Cls}$ for the association of dog ownership and time to initiation of medication for hypertension, dyslipidaemia and type 2 diabetes.

dog breeds' breed owners reported a high level of physical activity versus $52.3 \%$ in non-dog owners and $58.6 \%$ for mixed-breed dog owners. ${ }^{32}$ We chose to analyse these four 'active dog breeds' together (terriers, pointing, scent hounds and retrievers) to explore the association with CVD risk factors as they were all associated with lower risk of incident CVD events in our previous study, ${ }^{3}$ but should

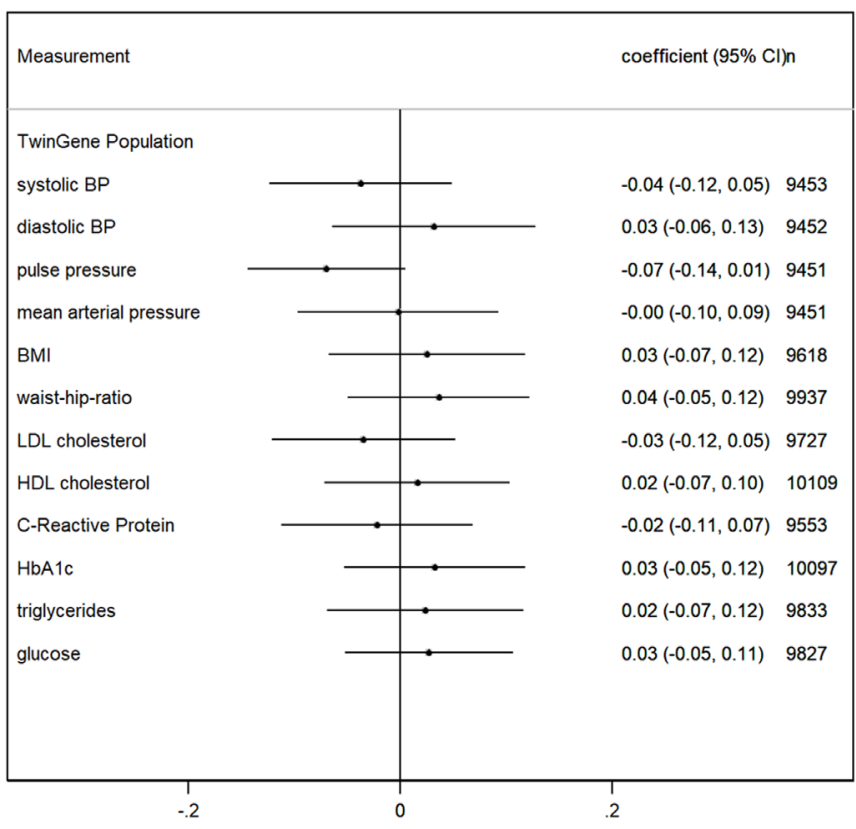

Figure 2 Coefficients and 95\% Cls for the exposure to dog ownership compared with non-dog ownership on SDtransformed biochemical and clinical measurements in the TwinGene. BMI, body mass index; BP, blood pressure; HDL, high-density lipoprotein; LDL, low-density lipoprotein. not be viewed as the only active breed groups in the study. We also note that the proportion of highest education level in the mixed-breed group was remarkably lower than the general population (20.9\% vs $29.3 \%)$. Although we adjusted for educational level, it is likely that there is unmeasured confounding from differences in healthseeking behaviour, smoking habits or stress in dog owners in working age groups. In TwinGene, we noted that additional adjustment for employment status (unemployed, retired, sick leave or unemployed) and a comorbidity index (for diseases other than CVD) were important confounders lowering the estimates. These covariates were not available in the national cohort, implying that the results in the national cohort are likely to have been confounded by these or other factors.

Our findings in TwinGene are different from an Australian cohort study in 5741 individuals with $13.6 \%$ pet ownership who found lower levels of plasma cholesterol, triglycerides and systolic blood pressures in pet-owners than non-owners. ${ }^{20}$ Dog owners $(6.3 \%)$ had better self-rated health but no difference in blood pressure than non-pet owners in cross-sectional analysis of the Nord-Trondelag Health Study (HUNT)-3 study $(\mathrm{n}=12297){ }^{13}$

There are a limited number of studies of the association between dog ownership and the risk of type 2 diabetes. A study by Lentino et al, ${ }^{16}(\mathrm{n}=916)$ showed that regular $\mathrm{dog}$ walkers $(\mathrm{n}=399,44 \%)$ in a primarily well-educated Caucasian population had lower BMI and were at lower risk of both dyslipidaemia and type 2 diabetes than other study participants. These findings were contradicted by Wright et $a l,{ }^{19}$ who showed that $\operatorname{dog}$ owners were more likely to be overweight, and have diabetes than non-owners in a study of 1179 community dwellers with $30 \%$ pet ownership. Differences in findings across countries could be due to differences in study design, or to inherent differences in dog management and the type of dog breeds in the country.

A previous study in this population showed a lower risk of cardiovascular disease and all-cause mortality in dog owners. ${ }^{3}$ The current study suggests that it is unlikely that hypertension and dyslipidaemia mediates these effects. Other potential factors that may explain this reduction in mortality include increased social well-being and decreased psychological stress. ${ }^{33}$

\section{Strengths and weaknesses}

The main strengths of our study include its size and the population-based approach increasing generalisability beyond healthy volunteers in a cohort study. To the best of our knowledge, this is the largest register-based study to date to explore the association between dog ownership and cardiovascular risk factors. At the same time, while national registers allow for large and unselected populations with no loss to follow-up, they lack information on individual attributes such as body mass index, blood pressure, lipid levels and physical activity. A strength of this study is that we were able to include 
additional clinical health measurements and socioeconomic variables using data from the TwinGene study supporting the presence of additional confounding of the relationship between dog ownership and cardiovascular risk factors from employment status and non-CVD comorbidities. Although our findings show an association between certain dog breeds and cardiovascular risk factors, these observational results do not imply a causal relationship.

The main limitation of the study is the possibility of remaining unmeasured confounding by unmeasured socioeconomic factors or pre-existing personality traits. Further, the register-based nature of our study made it impossible for us to account for pet-associated factors such as primary pet responsibility, physical activity, the level of dog attachment or indeed the reason for acquiring a dog. Physical activity related to dog walking may however be a mediator of the association between dog ownership and health outcomes and separating activity performed in relation to dog walking and other types of activity would be important. However, a large randomised study of dog ownership over several years cannot be done. Further, despite adjustment for several health, socioeconomic and lifestyle indicators, there is still a possibility of residual confounding or reverse causation. For instance, we could not assess health status before pet acquisition in the national cohort. A smaller study population, although not selected in relation to exposure or outcome, and possible misclassification of dog ownership (due to a lack of information on partners' dog ownership) or lifestyle questionnaire data (collected some years earlier) were important limitations in the subcohort analyses. Misclassification of dog ownership was also possible in non-married cohabiting partners without children in common as these would not be registered as cohabiting in the Register of The Total Population. Another important limitation is that we were unable to account for those that did not initiate treatment due to any of the three conditions. The Prescribed Drug Register does not keep a record of adherence to treatment or records of those prescribed lifestyle interventions such as diet or exercise.

\section{CONCLUSION}

In this large cohort study, we observed that dog ownership was associated with a minimally higher risk of initiation of treatment for hypertension and dyslipidaemia, and that ownership of dogs of the previously identified 'active dog breeds' was associated with a lower risk of initiating treatment for diabetes. These observations may suffer from residual confounding despite access to multiple important covariates, and future studies may add valuable information. The observed inverse association of $\operatorname{dog}$ ownership and cardiovascular disease previously reported in this population are unlikely to be explained by reduced hypertension and dyslipidaemia.
Author affiliations

${ }^{1}$ Department of Medical Sciences, Molecular Epidemiology and Science for Life Laboratory, Uppsala University, Uppsala, Sweden

${ }^{2}$ Department of Surgical Sciences, Orthopedics, Uppsala University, Uppsala Universitet, Uppsala, Sweden

${ }^{3}$ Division of Ruminant Medicine and Veterinary Epidemiology, Department of Clinical Sciences, Swedish University of Agricultural Science, Uppsala, Sweden

${ }^{4}$ Department of Medical Sciences, Clinical Epidemiology, Uppsala University, Uppsala, Sweden

${ }^{5}$ Department of Medical Epidemiology and Biostatistics, Karolinska Institutet, Stockholm, Sweden

${ }^{6}$ Division of Cardiovascular Medicine, Department of Medicine, Stanford University School of Medicine, Stanford University, Stanford, California, USA

Acknowledgements We acknowledge The Swedish Twin Registry for access to data. We would also like to acknowledge the Swedish Kennel Club and the National Board of Agriculture for granting access to the dog registers. They were not involved in any part of the study design, analysis, data interpretation, manuscript preparation or approval. Support by BILS (Bioinformatics Infrastructure for Life Sciences) is gratefully acknowledged. There was no compensation received for this assistance.

Contributors TF conceived the study and acquired funding. MM, AE, El, JS and LB contributed to the design of the study. TF acquired the national data and PKEM is responsible for the Swedish Twin Registry data. MM performed data cleaning. MM and TF ran statistical analyses. MM drafted the manuscript and all authors reviewed the manuscript.

Funding The study was funded by the Agria Research Foundation and the Swedish Research Council for Environment, Agricultural Sciences and Spatial Planning (FORMAS), grant number 2013-1673. TF has personal funding from the Goran Gustafsson foundation. The Swedish Twin Registry is managed by Karolinska Institutet and receives funding through the Swedish Research Council under the grant no 2017-00641.

Competing interests El is a scientific advisor for Precision Wellness and Olink Proteomics for work unrelated to the present project.

Patient consent for publication Not required.

Ethics approval The regional ethical review board in Stockholm, Sweden, approved the study (national study: 2012/1114-31/2, with amendment 2013-168732; TwinGene: 2007/644-31/2 and 2016/1392-31/1).

Provenance and peer review Not commissioned; externally peer reviewed.

Data sharing statement The register data that support the findings of this study were made available by record-linkage with data from Statistics Sweden, the National Board of Health and Welfare, the Swedish Kennel Club, Swedish Board of Agriculture and the Swedish Twin Register. Restrictions apply to the availability of these data, which were used under license and ethical approval for the current study, and so are not publicly available. Data are however available from the authors upon reasonable request and with permission of the Regional Ethical Review Board in Stockholm, Sweden.

Open access This is an open access article distributed in accordance with the Creative Commons Attribution Non Commercial (CC BY-NC 4.0) license, which permits others to distribute, remix, adapt, build upon this work non-commercially, and license their derivative works on different terms, provided the original work is properly cited, appropriate credit is given, any changes made indicated, and the use is non-commercial. See: http://creativecommons.org/licenses/by-nc/4.0/.

\section{REFERENCES}

1. Levine GN, Allen K, Braun LT, et al. Pet ownership and cardiovascular risk: a scientific statement from the American Heart Association. Circulation 2013;127:2353-63.

2. McNicholas J, Gilbey A, Rennie A, et al. Pet ownership and human health: a brief review of evidence and issues. BMJ 2005;331:1252-4

3. Mubanga M, Byberg L, Nowak C, et al. Dog ownership and the risk of cardiovascular disease and death - a nationwide cohort study. Sci Rep 2017;7:15821.

4. Yabroff KR, Troiano RP, Berrigan D. Walking the dog: is pet ownership associated with physical activity in California? J Phys Act Health 2008;5:216-28.

5. Westgarth C, Christley RM, Marvin G, et al. I Walk My Dog Because It Makes Me Happy: A Qualitative Study to Understand Why Dogs 
Motivate Walking and Improved Health. Int J Environ Res Public Health 2017;14:E936.

6. McConnell AR, Brown CM, Shoda TM, et al. Friends with benefits: on the positive consequences of pet ownership. J Pers Soc Psychol 2011;101:1239-52.

7. Venkatasamy VV, Pericherla S, Manthuruthil S, et al. Effect of physical activity on insulin resistance, inflammation and oxidative stress in diabetes mellitus. J Clin Diagn Res 2013;7:1764-6.

8. Roberts CK, Hevener AL, Barnard RJ. Metabolic syndrome and insulin resistance: underlying causes and modification by exercise training. Compr Physiol 2013;3:1-58.

9. Müllersdorf M, Granström F, Sahlqvist L, et al. Aspects of health, physical/leisure activities, work and socio-demographics associated with pet ownership in Sweden. Scand J Public Health 2010;38:53-63.

10. Statistica. Dog or cat ownership rates in households by race/ ethnicity in the United States in 2011. Google Scholar 2011

11. Wood L, Bosch D, Bulsara M, et al. More Than a Furry Companion: The Ripple Effect of Companion Animals on Neighborhood Interactions and Sense of Community. Soc Anim 2007;15:43-56.

12. Siegel JM, Angulo FJ, Detels R, et al. AIDS diagnosis and depression in the Multicenter AIDS Cohort Study: The ameliorating impact of pet ownership. AIDS Care 1999:11:157-70.

13. Enmarker I, Hellzén O, Ekker $\mathrm{K}$, et al. Health in older cat and dog owners: The Nord-Trondelag Health Study (HUNT)-3 study. Scand J Public Health 2012;40:718-24.

14. Friedmann E, Thomas SA, Son H, et al. Pet's presence and owner's blood pressures during the daily lives of pet owners with pre- to mild hypertension. Anthrozoös 2013;26:535-50.

15. Hoerster KD, Mayer JA, Sallis JF, et al. Dog walking: its association with physical activity guideline adherence and its correlates. Prev Med 2011;52:33-8.

16. Lentino C, Visek AJ, McDonnell K, et al. Dog walking is associated with a favorable risk profile independent of moderate to high volume of physical activity. J Phys Act Health 2012;9:414-20.

17. Parslow RA, Jorm AF. Pet ownership and risk factors for cardiovascular disease: another look. Med J Aust 2003;179:466-8.

18. Utz RL. Walking the dog: the effect of pet ownership on human health and health behaviors. Soc Indic Res 2014:116:327-39.
19. Wright JD, Kritz-Silverstein D, Morton DJ, et al. Pet ownership and blood pressure in old age. Epidemiology 2007;18:613-8.

20. Anderson WP, Reid CM, Jennings GL. Pet ownership and risk factors for cardiovascular disease. Med J Aust 1992;157:298-301.

21. Leslie BE, Meek AH, Kawash GF, et al. An epidemiological investigation of pet ownership in Ontario. Can Vet J 1994;35:218-22.

22. Flint EL, Minot EO, Perry PE, et al. Characteristics of adult dog owners in New Zealand. N Z Vet $J$ 2010;58:69-73.

23. Murray JK, Browne WJ, Roberts MA, et al. Number and ownership profiles of cats and dogs in the UK. Vet Rec 2010;166:163-8.

24. Downes M, Canty MJ, More SJ. Demography of the pet dog and cat population on the island of Ireland and human factors influencing pet ownership. Prev Vet Med 2009;92(1-2):140-9.

25. Westgarth C, Pinchbeck GL, Bradshaw JW, et al. Factors associated with dog ownership and contact with dogs in a UK community. BMC Vet Res 2007;3:5.

26. D'Agostino RB, Vasan RS, Pencina MJ, et al. General cardiovascular risk profile for use in primary care: the Framingham Heart Study. Circulation 2008;117:E86.

27. Lichtenstein P, De Faire U, Floderus B, et al. The Swedish Twin Registry: a unique resource for clinical, epidemiological and genetic studies. J Intern Med 2002;252:184-205.

28. Magnusson PK, Almqvist C, Rahman I, et al. The Swedish Twin Registry: establishment of a biobank and other recent developments. Twin Res Hum Genet 2013;16:317-29.

29. Hoffmann R, Lokrantz M, Lagerkvist C-J, et al. Värdet av hundar och katter i Sverige. 2017.

30. Delhey J, Newton K. Predicting Cross-National Levels of Social Trust: Global Pattern or Nordic Exceptionalism? European Sociological Review 2005;21:311-27.

31. Agria Pet Insurance Report. 2017. https://www.agria.se/globalassets/ sv/villkor/english/agria_villkor_hund_a4_aug2017_se_en_170807_v1. pdf

32. Pickup E, German AJ, Blackwell E, et al. Variation in activity levels amongst dogs of different breeds: results of a large online survey of dog owners from the UK. J Nutr Sci 2017;6:e10

33. Compare A, Zarbo C, Manzoni GM, et al. Social support, depression, and heart disease: a ten year literature review. Front Psychol 2013;4:384 